\title{
GLAD!
}

Revue sur le langage, le genre, les sexualités

11 | 2021

Archives, genre, sexualités, discours

\section{Provoquer des archives et invoquer la mémoire}

Plaidoyer pour (re)constituer le matrimoine du neuvième art français

Provoking Archives and Invoking Memory

\section{Marys Renné Hertiman}

\section{(2) OpenEdition}

Journals

Édition électronique

URL : https://journals.openedition.org/glad/3272

ISSN : 2551-0819

Éditeur

Association GSL

Référence électronique

Marys Renné Hertiman, « Provoquer des archives et invoquer la mémoire », GLAD! [En ligne], 11 | 2021, mis en ligne le 20 décembre 2021, consulté le 29 janvier 2022. URL : http://journals.openedition.org/ glad/3272

Ce document a été généré automatiquement le 29 janvier 2022.

\section{(i) $९$

La revue GLAD! est mise à disposition selon les termes de la Licence Creative Commons Attribution Pas d'Utilisation Commerciale - Pas de Modification 4.0 International. 


\section{Provoquer des archives et invoquer la mémoire}

Plaidoyer pour (re)constituer le matrimoine du neuvième art français

Provoking Archives and Invoking Memory

Marys Renné Hertiman

\section{Introduction : Absentes ? À propos de la rareté des créatrices de BD}

1 L'histoire de la bande dessinée est assez récente, puisque ce médium n'existe que depuis le XIX $\mathrm{XIècle}^{\mathrm{e}}$ quand le Suisse Rodolphe Töpffer publie sa littérature en estampes. Dans sa forme actuelle (art séquentiel reliant texte [souvent dans des phylactères] et images), la présence des femmes ayant œuvré dans la production de bandes dessinées n'a quasiment pas donné lieu à des recherches en histoire, en sociologie, ni en matière journalistique. Leur absence présumée et même soulignée par les acteurs de cette industrie, comme c'est le cas du Délégué général du festival d'Angoulême pour qui, et ce même en 2016, les femmes dans l'histoire de la BD peuvent se compter sur les doigts d'une main ${ }^{1}$. Pourtant, une requête approfondie des catalogues des maisons d'édition, d'archives et des sites spécialisés m'a permis de trouver plus de 620 créatrices ayant publié au moins deux bandes dessinées (strips, périodiques ou albums) dont certaines depuis 1900 (Madeleine-Amélie Dauphin). Si l'histoire du médium n'a pas encore révélé son matrimoine, il n'en demeure pas moins pertinent de s'interroger sur la manière dont les institutions légitimantes semblent ignorer la prolifique production des créatrices contemporaines telles Elsa Abderhamani, Théa Rojzman, Marguerite Abouet, Karina Lyfoung, Aude Picault, Carol Maurel, Reine Dibussi, Valp, Mélaka, Marie Bardiaux-Vaïente, Yoon-Sun Park, Sandrine Revel, Véro Cazot, Jenny Rakotomamonjy, Tédone, Christelle Pécout, Sophie Chaumard, Vanyda, Tanxx, ou encore Algésiras. 
2 Les lacunes concernant la participation des femmes dans le neuvième art se constatent également dans le cadre de la formation des futur.e.s bédéastes elleux-mêmes. Comme l'a constaté Maëlys Tirehote-Corbin (2019), si les autrices représentent environ $27 \%$ de la population des bédéastes en France, seulement 5 à 7 d'entre elles sont inscrites dans les livres de $\mathrm{BD}^{2}$. En dehors des rapports de pouvoir propres à la construction d'une histoire genrée, comment interroger cette absence présumée de créatrices ? De même, comment saisir la richesse de pratiques, de savoirs et de savoir-faire dans cette industrie sans considérer la transmission et la conservation d'archives des créatrices elles-mêmes ? Enfin, comment produire un savoir sur cette industrie, en se contentant seulement de quelques sources historiques sans les explorer ni les contester?

3 Questionner la rareté des travaux à ce sujet, dans le domaine académique, implique de (re)mettre en lumière un problème structurel non résolu, relatif aux rapports de pouvoir genrés, et ce même au sein des espaces dits subversifs. La bande dessinée n'est plus un champ illégitime ni marginal en France et la théorisation du médium est une constante. Les problématiques les plus récurrentes vont se centrer sur les aspects artistiques, sémiotiques ou historiques de ce champ, et sont généralement observées à partir d'un registre ou d'un air culturel particulier. Pourtant, son étude depuis une perspective de genre ou dans le cadre d'une approche féministe, n'a quasiment pas été développée. À l'exception de quelques travaux ${ }^{3}$, l'œuvre et le rôle des autrices sont des objets qui semblent ne pas attirer l'attention de la recherche institutionnelle française. Cette situation renforce l'impression d'un champ culturel à part entière, mais dépourvu de son matrimoine.

4 Puisqu'elles sont très peu représentées dans la sphère publique, s'intéresser aux créatrices de bande dessinée (scénaristes, dessinatrices, coloristes) nécessite un effort réel. Le manque de sources complique tout travail sérieux et approfondi autour d'elles. Un des risques permanents liés à cette situation concerne l'abandon de certains sujets d'étude en raison du manque de documentation ou des difficultés d'accès au terrain. Or, si la littérature grise (presse spécialisée notamment), a commencé à combler certaines lacunes, et ce dès l'essor du médium en France ${ }^{4}$, dans le domaine académique, les études qui questionnent le travail, le genre et les relations de pouvoir ou le rôle des femmes dans cette industrie semblent inexistantes.

5 La thèse de Jessica Kohn, soutenue en 2018, est l'un des travaux de contextualisation les plus aboutis à ce sujet. Cette étude, qui part d'une approche prosopographique, met en lumière les noms des premières dessinatrices en France et en Belgique. Sa méthode de recherche (entretiens semi-dirigés et travail d'archives), très utile pour retracer les premier.e.s professionnel.le.s du secteur, ainsi que la naissance d'un métier, offre des perspectives pour de nouveaux travaux sur la BD impliquant une dimension genrée. Il en va de même pour la recherche de Florie Boy (2009), qui permet de situer une mutualisation discursive, c'est-à-dire un discours qui peut être commun à un groupe donné, entre les créatrices au fil des années (dès 1970 aux années 2000). Une approche historique similaire, avec un corpus presque identique, a également été développée dans le mémoire de master de Bérénice Motais de Narbonne (2016) et dans les travaux de fin d'études de Virginie Talet (2004) et de Blanche Delaborde (2005), tous deux sur la revue Ah! Nana. À l'exception des recherches de Jessica Kohn, de Pierre Nocérino ${ }^{5}$ et de Maëlys Tirehote-Corbin ${ }^{6}$, les corpus académiques s'intéressent aux mêmes professionnelles, celles et ceux qui ont contribué dans Ah! Nana ou Métal Hurlant, et celles qui apparaissent dans les quelques (et brèves) notices de dictionnaires et dans les 
ouvrages spécialisés?. Quant à une réflexion genrée sur la bande dessinée, la revue Alternatives francophones, avec son dossier sur "La bande dessinée au féminin " publié en 2016, se veut la première (Milquet \& Reyns-Chikuma 2016:02) à aborder ce sujet dans le monde académique francophone. Ce numéro s'intéresse à la situation des créatrices francophones et à leur évolution en tant que groupe social, mais aussi à la production contemporaine, tout en proposant de nouvelles perspectives sur la signification artistique et sociale de cette production dite au féminin. Enfin, depuis mars 2020, avec un groupe de chercheuses appelées les Bréchoises ${ }^{8}$, on tente de mettre en lumière le matrimoine de la bande dessinée française, en intégrant une approche féministe qui mobilise des méthodes issues des études de genre, des théories de l'intersectionnalité et du point de vue.

6 Cet article porte sur un qui vise à provoquer des archives pour en faire des monuments voulus, organisant la transmission; et des monuments-mémoire, conjurant l(oubli (d'après Florence Descamps, 2019: 113). Présentant ainsi les étapes d'un projet aux enjeux politiques et scientifiques, cet article, qui porte donc sur les traces du matrimoine de la bande dessinée, se structure de la manière suivante: dans un premier temps, je vais présenter les coulisses de ce projet archivistique. Et, dans un second temps, je vais revenir sur une terminologie qui, appliquée à l'archivistique, permettrait de donner un nouveau sens à l'écriture de l'histoire du neuvième art français. Cet article propose ainsi une réflexion sur la mise en mémoire des créatrices de BD en France, catégorie sociale constamment confrontée au manque de reconnaissance, aux discriminations et à l'invisibilisation, voire à l'exclusion.

\section{Provoquer les traces}

\section{Conception du séminaire « Matrimoine de la BD »}

7 Les seules enquêtes conduites à propos de la population des bédéastes en France font uniquement référence aux artistes-auteurices, c'est-à-dire, celleux qui apposent leur signature et perçoivent des droits d'auteurice'. Ainsi, l'insuffisance des sources et d'informations concernant les créatrices de bande dessinée en France, a conduit à un chiffrement inexact du nombre de professionnelles exerçant dans la période contemporaine : 12 \% d'autrices d'après le rapport Ratier de l'Association des Critiques de BD (ACBD, 2015), 27 \% d'après les États Généraux de la BD (ÉGBD, 2016). Les ouvrages spécialisés ne retiennent également qu'une part infime du nombre de créatrices de $\mathrm{BD}^{10}$. Pourtant, j'ai déjà listé plus de 620 créatrices (autrices complètes, scénaristes et coloristes) ${ }^{11}$, parmi lesquelles des femmes ayant travaillé durant la première moitié du XXe siècle : Davine, Paulette Blonay, Marie-Hélène Sigaut, Marianne Clouzot, Claudine Conin, Madeleine-Amélie Dauphin, Françoise Ménager, Anne Chatel, Yolanda Canale, Natalie d'Arbeloff, Joëlle Savey, Viviane Nicaise, Françoise Alto-Bianci et bien d'autres.

8 La disparité concernant les chiffres autour d'une bande dessinée faite par des femmes illustre quelques-unes des inégalités dans cette industrie et plus largement l'absence d'un travail de fond sur ces acteurices. Ce contexte on peut le comprendre à partir de l'étude par Christine Détrez (2018: 159-160), qui expliquait que l'exclusion et l'invisibilisation vécues par les professionnelles de la culture, se sont produites en trois manches : la première interdisant l'accès aux femmes à un certain domaine, la seconde niant ou minorisant leur existence et leur rôle dans ledit domaine et la troisième 
dévalorisant le travail qu'elles exercent ou ont exercé. Ces trois manches permettent de questionner les lacunes de l'histoire culturelle face à un matrimoine qui paraît inexistant. S'en saisir, pour produire de nouvelles archives et d'autres sources documentaires, justifie l'importance et la pertinence de s'intéresser à un réservoir d'histoire largement ignoré ${ }^{12}$.

9 C'est à partir de cette problématique que s'est construit le projet «Créatrices de bandes dessinées : histoire, mémoire, revendications et représentations des femmes dans le neuvième art », coordonné par les Bréchoises. Son principal objectif : donner la parole aux créatrices et aux actrices de la recherche universitaire émergente sur le sujet, pour cimenter le matrimoine de la BD. Plusieurs missions ont été programmées à l'intérieur de ce projet qui vise, de manière plus vaste, la visibilisation et la valorisation du parcours des créatrices qui opèrent individuellement et/ou collectivement. L'une de ces missions consiste en la constitution d'un fonds d'archives consacré aux créatrices contemporaines exerçant en France. Il sera créé à partir du séminaire « Matrimoine de la $\mathrm{BD}$ ", dont la première édition qui devait avoir lieu à la MSH Paris Nord durant l'année universitaire 2020-2021, n'a commencé qu'en octobre 2021 en raison du contexte sanitaire liée à la pandémie du CoVID19. L'ingénierie d'un tel projet, nécessitait de l'accompagnement et un soutien institutionnel important, sans quoi, il n'aurait pas pu s'imaginer. Dévoiler les coulisses d'un projet, encore en chantier, est assez inhabituel. Toutefois, l'important travail réalisé en amont peut ouvrir des perspectives pour des projets portant sur la mise en mémoire d'autres matrimoines ou d'autres groupes invisibilisés.

\section{La conception du projet}

10 Le projet «Créatrices de bandes dessinées: histoire, mémoire, revendications et représentations des femmes dans le neuvième art » ne répond pas à un appel d'offres ni à une commande institutionnelle, mais bien à un besoin scientifique, historique, social et culturel. Partant de mes difficultés à obtenir des sources documentaires (outre les témoignages des créatrices elles-mêmes), en mars 2020 j'ai lancé l'idée de la création d'un groupe de travail portant sur les femmes dans la BD, au sein de l'association La Brèche ${ }^{13}$, dont je fais partie. Suite aux premières réunions et aux discussions aboutissant au même constat (toujours les lacunes et les discriminations de l'industrie face aux femmes qui l'ont construite), naissent Les Bréchoises. Trois volets sont articulés à l'intérieur de leur projet: un atelier séminarial, un colloque et une exposition (dans le cadre de cet article, il sera seulement question du premier volet ${ }^{14}$ ). $\mathrm{Au}$ vu des pratiques et des disciplines de chacune des personnes impliquées dans le groupe de travail (sociologie, arts et histoire de l'art principalement), la réflexion de la méthode anthropographique s'est vite dessinée, afin de tenter de combler cette présumée absence d'une histoire des femmes bédéastes. Nous avons ainsi établi nos objectifs (de visibilisation et de valorisation), l'esquisse de corpus que nous voulions constituer (sources matérielles et immatérielles), nos différents besoins (humains, logistiques, financiers et spatio-temporels) et les différentes formes d'aboutissement du projet (atelier séminarial conduisant à la constitution d'un fonds d'archives, et pouvant être valorisé auprès du grand public). 


\section{Le cahier des charges}

11 La faisabilité d'un projet de visibilisation, qui implique une vaste opération de mise en mémoire, comprend différentes phases devant être inscrites au cahier des charges. Tout d'abord, il est nécessaire d'assurer l'accès et le maintien au terrain (ici les réseaux de créatrices). Partir du terrain est essentiel pour concevoir un projet avec les personnes concernées, et non pas en spéculant sur leurs éventuels besoins, quitte à faire des concessions avec les protocoles scientifiques d'une enquête ${ }^{15}$. Ensuite, pour établir et contractualiser nos différents partenariats, nous avons initialement contacté nos collègues et institutions de rattachement pour leur présenter le projet. Durant cette phase, nous avons obtenu les informations concernant les appels à projets (pour financer le projet ${ }^{16}$ ), et avons constitué le comité scientifique qui nous accompagne.

12 Veillant à répondre aux calendriers des trois appels à projets, nous avons entamé la phase de rédaction de nos dossiers de candidature. Cette phase était assez intense et nécessitait une validation sur plusieurs niveaux (d'abord par le comité d'organisation, ensuite en collaboration avec le comité scientifique et après, avec les chargé.e.s du service de valorisation des institutions ayant lancé les appels). Nous avons convoqué une série de créatrices $^{17}$ dès la contractualisation des premiers partenariats, notamment ceux liés aux lieux de conservation et de valorisation de leurs archives (Archives du féminisme et Archives départementales de l'Ille-et-Vilaine). Cette convocation de créatrices ne s'est pas faite sans difficulté, et malgré une certaine confiance instaurée avec elles et leur conscience de l'importance de ce projet de mise en mémoire, les témoins et acteurices de l'histoire ne veulent pas toustes être des sources. Parmi les arguments donnés, les phénomènes de reconnaissance et de légitimité semblent conditionner une partie des refus d'entrée dans le projet. D'autres, au contraire, ont souligné les difficultés à se plonger dans toute une vie, en raison de leur pudeur ou de la douleur que pouvait impliquer le fait de revenir sur certains aspects de leur vie ${ }^{18}$. Enfin, dans un espace où on les renvoie constamment à leur statut minoritaire, comment considérer leur parcours comme suffisamment pertinent pour illustrer ou représenter un groupe donné ? Dès lors, nous remarquions la fragilité, la complexité et la sensibilité du travail à partir des archives personnelles, car « à quelles conditions et sous quel rapport, la parole d'une personne peut devenir le patrimoine [sic] des autres?» (Hiraux \& Mirguet 2013 : 19). En effet, c'est dans ces résistances que résident aussi les paradoxes du travail avec les archives (orales notamment) et certaines de leurs producteurices.

\section{Collecte d'archives matérielles et immatérielles}

13 L'aboutissement de ce projet concerne la conception d'un fonds d'archives, composé par des sources matérielles et des témoignages filmés. Cette collecte se fait à double temps: d'abord, par des réunions avec les créatrices, afin de définir ensemble les sources qu'elles voudront bien donner ou déposer. Ce temps d'échange permet aussi de compléter un calendrier de vie et de parfaire le canevas d'entretien, tous deux conduisant à l'élaboration de l'entretien filmé qui sera produit ultérieurement. Ensuite, la deuxième partie, organisée sous la forme d'un atelier séminarial, va présenter des interventions mettant en lumière l'histoire de la BD à partir de l'inclusion des femmes dans cette industrie, puis va conduire à la collecte des témoignages de créatrices convoquées, à partir d'un entretien semi-dirigé (filmé). Provoquer ces sources orales, 
c'est-à-dire conçues dans une volonté de sauvegarde matrimoniale, est aussi important pour restituer les voix, les gestes et les visages de ces créatrices, ce qui s'entend comme un moyen supplémentaire pour leur inscription dans la postérité. Avec cette collecte, nous revendiquons la valeur mémorielle des créatrices de BD.

\section{Des archives orales au matrimoine de la BD}

Dans l'intérêt de consolider la conception de ce projet, j'ai suivi des séminaires (notamment celui de Florences Descamps sur les archives orales) et différents événements scientifiques (journées d'étude et conférences). Cela m'a permis de découvrir le jargon de toute une pratique et de mieux comprendre les points de jonction et les spécificités de l'histoire, comme discipline, vis-à-vis de la sociologie ou de la pratique empirique dans les SIC. Avec cette démarche autodidacte, je voulais aussi avoir d'autres outils pour réfléchir à l'effacement du matrimoine de la BD et l'occultation des femmes qui l'ont tissé. Considérer l'histoire de ce médium telle qu'on la connaît aujourd'hui, c'est-à-dire uniquement au travers des traces partiales et partielles, c'est ignorer que «la vérité dogmatique consiste à effacer de l'écrit sa trace d'histoire » (Legendre, cité par Favret-Saada, 2020 [1977] : 21). Alors, pour comprendre le neuvième art, il faut aussi partir des récits et des témoignages de ses acteurices. Étant donné que la parole des femmes y est absente, l'intégrer sans parler à leur place est un acte aussi scientifique que politique. De ce fait, «la création d'archives orales apparaît comme un moyen facile de rassembler des matériaux pour entreprendre ou nourrir leur propre histoire » (Descamps 2019 : 67). Je me répète, mais c'est parce qu'il me paraît nécessaire d'insister sur l'évincement d'un matrimoine qui existe pourtant bel et bien puisque, malgré les plus des 600 créatrices qui ont œuvré dans l'industrie de la BD française dès 1930, seule une poignée est inscrite dans quelques notices des dictionnaires et des livres sur l'histoire de la bande dessinée. Aujourd'hui, il n'y a même pas de monographie sur Claire Brétecher, dont le nom revient constamment sur les notices de ces ouvrages spécialisés et qui a une présence très importante dans l'espace médiatique. Par ailleurs, si des mémoires de recherche parlent de l'œuvre de certaines créatrices comme Chantal Montellier, Jeanne Puchol, Marjane Satrapi, Florence Cestac ou Jul Maroh ${ }^{19}$, ces études ne problématisent pas les conditions de vie, de travail ni le rôle de ces créateurices dans cette industrie. L'ambition de ce projet de matrimonialisation est donc d'intégrer cette mémoire dans la sphère publique, de la conserver et de la valoriser afin de réparer l'oubli et le manque de reconnaissance auxquels sont constamment confrontées les créatrices de BD.

J'insiste, malgré les quelques études à leur sujet (je retiens notamment la thèse de Jessica Kohn) l'inaccessibilité du grand public à ces travaux (car destinés à un public académique et disponibles dans des bibliothèques universitaires) rend fragile le maintien des femmes dans l'histoire culturelle contemporaine. Pour une inscription dans la mémoire collective, il faut des publications généralistes qui traitent aussi des professionnelles de la BD. Or, comme disait Isabelle Bastien-Dupleix, commissaire de la rétrospective de Claire Bretécher à la BpI en 2016, «il n'y a pas de lien entre la notoriété des femmes et [leurs] traces dans l'histoire " (Hertiman, 2020), expliquant par-là les difficultés rencontrées pour construire une exposition à son sujet. Le besoin de mise en mémoire des femmes (et d'une histoire genrée), dans la bande dessinée n'est plus un constat à faire et "à cet égard, les archives orales s'apparentent à une 
démarche de réappropriation et de "reconnaissance" du passé et de tous ceux qui ont précédé » (Descamps, 2019 : 73).

\section{Penser le manque d'archives et l'effort de conservation, à partir d'une terminologie permettant de (ré)écrire l'histoire depuis une perspective politique}

Les travaux sur la mémoire, notamment à partir des projets d'archives, ont longtemps été attribués au domaine de l'histoire. Pourtant, le caractère trans-contributif de cette discipline est tangible : elle emprunte autant à la sociologie qu'aux sciences politiques et à l'ethnographie (Descamps, 2019), et l'oublier équivaudrait à ne pas dépasser les points aveugles qui ont pu la structurer. Ainsi, l'écriture de l'histoire (ou sa réécriture) suit un chemin lexicographique aussi varié qu'imagé lequel semble a priori accessible même pour les historien.ne.s sans titre. Parmi les personnes qui composent avec la terminologie de l'histoire sans être formellement inscrites dans cette discipline, on retrouve les créateurices de bande dessinée qui, dans la gestation de leur projet d'album (surtout s'il est inscrit dans un registre dit du réel), vont se documenter, faire l'état de l'art, observer, analyser, vérifier, récolter des données, voire en produire d'autres. Ayant observé ces pratiques, j'ai pu comprendre que, en dehors d'une éventuelle problématisation, ce n'est pas une routine si éloignée du travail mené par les chercheureuses. En effet, certains mots ayant servi à l'écriture de l'histoire (au sens large et polysémique de ce terme ${ }^{20}$ ), sont aussi employés par des créateurices de BD et par des chercheureuses en sciences humaines et sociales. Ainsi, nous tissons nos projets avec un vocabulaire qui se rejoint à plusieurs reprises : la dépouille, l'archive, l'histoire et la mémoire. Toutefois, le terme matrimoine, dont la signification concerne une mise en pratique transmissive, est rarement employé, surtout si ce n'est pas dans un cadre militant. Dès lors, questionner les termes employés dans une recherche (bédéiste ou scientifique), me permet de revoir le(s) sens qu'entoure(nt) ce(s) projet(s). De ce fait, pour restituer l'histoire des femmes dans la BD, il me paraît nécessaire de faire un point sur certains éléments du vocabulaire historiographique ${ }^{21}$.

\section{Archive}

17 Le Dictionnaire de terminologie archivistique, définit les archives comme l'ensemble de « documents, quels que soient leur date, leur forme et leur support matériel, produits ou reçus par toute personne physique ou morale, et par tout service ou organisme public ou privé, dans l'exercice de leur activité (Direction des Archives de France, 2007 [2002] : 10)». Prendre en compte cette acception, permet d'intégrer les traces expérientielles (celles qui rendent compte du vécu des individus), même celles des oublié.e.s de l'histoire. Pratiquer l'archive nécessite divers gestes et l'exploitation de certains outils, surtout ceux qui concernent la conservation des traces laissées ou à produire. Pourtant, il ne s'agit pas de prendre les archives comme un fond de stockage, où l'on puise des éléments qui peuvent éventuellement répondre à nos problématiques, mais bien d'un espace où, geste après geste, on tente de trouver des réponses aux questions qui émergent avec chaque trace repérée. D’ailleurs Arlette Farge précisait à ce sujet que, dans (et avec) les archives, on ne pouvait pas s'attendre à trouver ce que l'on venait expressément chercher : «il ne s'agit pas d'y découvrir, une fois pour toutes, 
un trésor enfoui, offert au plus malin ou au plus curieux, mais d'y voir un socle permettant à l'historien de rechercher d'autres formes du savoir qui manquent à la connaissance » (Farge 1997 : 70).

Quelle que soit sa forme (audiovisuelle, imprimée, sonore, orale, etc.) ou sa fonction (conservatoire, transmissive ou monumentaire ${ }^{22}$ ), les archives peuvent être constituées (pour répondre à un sujet donné), mais elles peuvent aussi se perdre ${ }^{23}$, se détruire, s'effacer, se commémorer ou s'inventer, se provoquer. L'état et le statut des archives sont des pistes pour évaluer (ou au moins y réfléchir) le rapport de pouvoir entre les groupes hégémoniques et subalternisés. À ce propos, José González et l'équipe du Centre d'études ibériques et ibéroaméricaines - cultures romanes et amérindiennes (CEIIBA), ayant initié un cycle d'études sur l'histoire de l'Amérique latine, ont insisté sur la dimension politique, voire ontologique, de l'archive ${ }^{24}$, en le désignant comme le :

premier lieu dans son renvoi au pouvoir et à l'autorité [...] qui rassemble les énoncés relevant d'un même positionnement, inséparables d'une mémoire et d'institutions qui leur confèrent leur autorité (Maingueneau 1991 et 2002). Cette articulation permet [de se demander] quels sont les a priori historiques qui ont rendu réels les énoncés ainsi diffusés et ont rendu impossibles d'autres énoncés trop hétérogènes » (González 2017 : s.p.).

Les archives répondent à une dimension normative (elles ont été constituées selon des normes et des contraintes spécifiques) qui émane de l'effet de réalité (ou de légitimité) procuré par sa source : un témoignage est-il considéré de la même manière qu'un rapport des faits ? Est-ce que le témoignage d'une créatrice de BD sera considéré de la même manière que le témoignage du Délégué Général du principal festival de BD en Europe ? Si cet effet peut se déconstruire pour continuer à saisir les autres sens (en imposant encore la nécessité d'autres questionnements), les énoncés qui les constituent vont acter la fonction performative du document. En effet, la lecture d'un document doit comprendre les codes et les normes qui l'ont conditionné et qui pèsent sur l'archiviste et/ou le producteur/la productrice d'archives au moment de leur création. Un exemple concret, qui m'éloigne de mon sujet, mais permet de rendre compte de la fonction performative du document à potentialité archivistique est le registre ou acte de naissance qui vient conditionner le genre de la personne qui vient d'être mise au monde. Pour ce qui est du champ de la bande dessinée, des sources signalant l'absence présumée des créatrices de BD (dictionnaires, études scientifiques, rapports, discours publics, etc.) vont conditionner l'imaginaire et les pratiques des créatrices ellesmêmes ${ }^{25}$. Il faut noter que le rôle de l'institution ayant produit l'archive est central pour légitimer ou renforcer sa performativité. Ainsi, les sources produites par le Festival d'Angoulême risquent d'avoir une plus grande influence que les sources produites par un amateur de bande dessinée, voire par des acteurices de cette industrie qui ne bénéficient pas d'une grande reconnaissance. Il en va de même pour la désignation du statut artistique des créateurices de BD, celle-ci passant, entre autres, par la source attestant qu'iels ont suivi une formation en Arts, ou par leurs contrats auprès des maisons d'édition ${ }^{26}$.

20 La pratique de l'archive chez ces artistes-auteurices ne passe pas que par les sources qui attestent de leur construction artistique et professionnelle. Elle passe aussi par son usage documentaire, voire sa reproduction réencadrée dans l'album, avec ou sans transformation, dans le texte et le hors-texte. Dans une conférence à la Sorbonne Nouvelle (2021), Markus Arnold, professeur de littérature française à l'université de Cape Town, observe trois usages dans la bande dessinée sériée au tournant post- 
colonial : d'abord, l'usage relatif à une restitution «fidèle " dans un souci mémoriel ; ensuite la quête de mémoire depuis une approche archéologique et, enfin, par le biais de l'empreinte autobiographique. L'archive en bande dessinée serait donc un gage de véracité et de légitimité, pour le récit bédéiste et pour la justification de la construction socioprofessionnelle de son auteurice.

\section{Dépouiller}

Pour Arlette Farge, le dépouillement est un «terme joliment évocateur - [qui] oblige à quantité de gestes, et l'opération intellectuelle décidée au départ, aussi complexe soitelle, ne peut en aucun cas les éviter" (Farge 1997: 71). Rappelant le geste d'une recherche pouvant être intrusive (on enlève chaque couche, chaque voile d'un document pour tenter de trouver ce que l'on cherche), le dépouillement peut conduire à un investissement émotionnel, corporel et temporel très important. Pour la création de son album Charonne Bou Khadir, Jeanne Puchol a puisé dans les archives familiales et de presse pour revenir sur la manifestation en faveur de l'indépendance de l'Algérie, le 8 février 1962, où neuf personnes ont perdu la vie à la rue de Charonne. Le temps long de l'archive et de la reconstitution de l'événement ont pris une place prépondérante dans le processus de création de cet album, qui croise documentation et récit autobiographique, et qui est l'une des œuvres les plus personnelles de l'autrice.

L'énumération des actrices de cette industrie passe également par le dépouillement très important de catalogues, d'ouvrages spécialisés ou des archives des maisons d'édition. Cette pratique vise à observer, de manière critique, ce que les autorités (ici l'industrie et les institutions culturelles) ont valorisé pour instaurer une mémoire, puis écrire une histoire. La thèse déjà citée de Jessica Kohn, illustre l'investissement très important des historien.ne.s qui souhaitent s'aventurer dans la quête de dessinatrices, ayant été parfois inscrites dans les registres des maisons d'édition ${ }^{27}$, mais rarement crédités dans les revues avant les années 1960. Plus qu'un inventaire, il s'agissait d'exhumer soigneusement trente-deux illustrés pour un corpus de 400 dessinateurices. Dans les annexes de sa thèse, Kohn nous explique que ce travail colossal a pu se réaliser grâce à d'autres gestes similaires, préalablement réalisés par un des sites gérés par Bernard Coulange :

L'existence d'un dépouillement préalable sur le site «bandes dessinées oubliées » a permis d'ouvrir cette liste à des illustrés à l'existence moins pérenne, mais qui ne firent pas moins partie du paysage éditorial des années concernées. Pour ne pas sous-estimer l'importance de la presse généraliste dans le développement de la profession de dessinateur-illustrateur, ce dépouillement a été complété par un sondage de quatre quotidiens proposant à la fois des bandes et des dessins de presse, à raison d'une semaine tous les ans pendant leur période de publication : France-Soir et L'Humanité en France ; Le Soir et Le Peuple en Belgique (Kohn 2018 : 685).

Le protocole de recherche de Jessica Kohn manifeste le temps long de l'archive. Un temps qui peut s'avérer fastidieux et frustrant. Pour ce qui concerne mon travail sur les autrices de $\mathrm{BD}$, le geste répétitif du comptage des listes d'albums vendus en France ${ }^{28}$, visant à suivre la production des femmes dans la BD, plus que chronophage, s'est avéré psychologiquement éprouvant: dans une pénurie des traces des femmes bédéastes, quand et sur quoi devais-je arrêter le dépouillement? Au moment de dépasser la période contemporaine, celle qui concerne mon étude? Mais, que faire de cette 
intuition qui dit que, dans l'histoire de cet objet médiatique récent, il y a des récits permettant de mieux comprendre le contexte de la praxis discursive ${ }^{29}$ des autrices d'aujourd'hui?

\section{Histoire et mémoire}

Le traçage des femmes qui ont contribué à ce qu'est la bande dessinée française aujourd'hui n'est pas une mince affaire. Dépouiller les archives demande du temps, mais que faire quand les traces sont imperceptibles ou demeurent trop hétérogènes? Déjà, partir du constat qu'il ne suffit pas d'inventorier, mais de porter un regard critique sur ces traces, pour ainsi interroger ce qui a trop souvent été négligé, l'expérience qui, n'allant "pas de soi, doit être regardée comme un objet d'analyse " (Scott, 2009 : 08) comme le précisait Joan W. Scott. Mais, comment saisir ou capter des expériences non documentées? Comment documenter ce qui semblait sans histoire et sans mémoire ? Pour explorer ces questions, il me semblait nécessaire de revenir sur la dyade histoire/mémoire, couple terminologique polémique ${ }^{30}$ et polysémique, qui permet de comprendre la manière dont les traces peuvent s'inscrire ou être effacées de la sphère publique.

Deux entrées de dictionnaire référencent l'Histoire comme la "connaissance et récit des évènements du passé jugés dignes de mémoire ; les faits ainsi relatés » (Le Robert), et comme la « recherche, connaissance, reconstruction du passé de l'humanité sous son aspect général ou sous des aspects particuliers, selon le lieu, l'époque, le point de vue choisi ; ensemble des faits, déroulement de ce passé » (CNRTL). Ces définitions insistent sur le caractère plastique du passé (car reconstruit, mis en récit), pouvant donc être modelées. Loin d'être neutre et dénouée de tout désintéressement, l'Histoire, reconstruite souvent par des sources archivistiques, est le fruit d'une sélection réfléchie et hiérarchisée. La valeur accordée par l'historien.ne au récit à établir est inextricablement liée au contexte socioculturel et aux normes juridiques, et d'historicité, de l'époque où ces récits sont construits.

Observer la mémoire comme source première de la reconstitution du passé est primordial, ne serait-ce que par cette polysémie où se manifeste la dimension politique et philosophique de sa valeur. Définie par le CNRTL comme la « faculté comparable à un champ mental dans lequel les souvenirs, proches ou lointains, sont enregistrés, conservés et restitués ", la mémoire adjuge aussi les traces laissées par un fait ou un individu « en vertu de ses talents, de ses qualités, de ses hauts faits ou à cause de ses méfaits ». Articuler la mémoire à la valeur accordée à des personnes, des objets ou des faits, relève du degré de connaissance et de reconnaissance qui leur seront attribuées.

La bande dessinée, dont la reconnaissance et la légitimité (Aquatias 2018) demeurent fragiles, a longtemps été considérée comme un art sans mémoire (Berthou 2011-2017). Les contraintes éditoriales du médium ${ }^{31}$ l'ont conduite à emprunter des circuits alternatifs qui éclatent sa mémoire ${ }^{32}$. Son histoire a été écrite à partir d'une production masculine, et les travaux des historien.ne.s se sont centrés notamment sur les maisons d'édition, sur certaines revues (À suivre, Ah! Nana, Métal Hurlant) ou sur des auteurs ayant une côte importante dans ce marché éditorial et instaurant son canon (Hergé, Wolinski). Cette mise en mémoire s'est donc faite en excluant les groupes structurellement minorés, comme les femmes ou les personnes racisées. De ce fait, on 
ne peut pas dire que la bande dessinée est un art sans mémoire, mais plutôt amnésique et oubliant son matrimoine.

\section{Matrimoine}

Les dictionnaires généralistes ainsi que la littérature grise renvoient le matrimoine au mariage (hétérosexuel notamment) et aux alliances institutionnelles. En dehors d'un cadre militant, l'utilisation du terme matrimoine se fait rarement ${ }^{33}$ pour référencer sa fonction transmissive, celle qui est liée à l'héritage, au legs, contrairement au patrimoine, dont la racine signale tout aussi bien la transmission qui se trace, mais cette fois-ci du côté du père (patri/pater = père ; monium $/$ munio = munir). Consciente et soucieuse de la rupture de transmission, je considère vital de questionner la constitution de l'héritage de la BD, partant déjà de cette terminologie genrée. Ainsi, on peut (re)construire de manière critique une démarche de mise en mémoire (et de monumentalisation) de l'héritage laissé par les créatrices en France.

En 2005, lors de la journée d'étude «La bande dessinée: un nouveau chantier pour l'histoire des femmes et du genre» (Mnémosyne 2005), l'association Mnémosyne plaidait pour commencer le chantier de l'histoire des femmes et du genre dans la BD. Pourtant, en dehors des quelques études mentionnées en introduction, le manque considérable de sources et de documents les concernant peut effrayer les chercheureuses, notamment dans le cadre des recherches non financées. C'est pourquoi les Bréchoises s'impliquent dans des démarches critiques et réflexives autour du rôle qu'ont joué (et que jouent encore), les femmes bédéastes. Mettant en commun leurs observations et leurs réseaux, elles s'engagent à consolider cette recherche à partir d'un regard féministe et d'une approche transdisciplinaire faisant appel aux comics studies, à l'histoire sociale et culturelle, aux gender et cultural studies, à l'anthropographie, aux sciences de l'information et de la communication (SIC), à la littérature, à l'histoire des Arts et à la sociologie. Avec leur projet de monumentalisation (ou de mise en mémoire), les Bréchoises inventent de nouvelles sources et archives et provoquent la restitution de celles existantes ${ }^{34}$. Il s'agit donc d'une démarche à la fois scientifique et militante, et en réclamant ce matrimoine, on met en lumière l'effacement des femmes dans l'histoire, et on signifie la prégnance politique de ce que certain.e.s veulent encore considérer comme un néologisme ${ }^{35}$.

\section{Conclusion}

31 L'expérience en cours, d'une recherche qui part d'un corpus autoconstitué visant à provoquer les traces du matrimoine du neuvième art français, me place dans une posture ambiguë, entre militantisme et engagement scientifique. La collecte d'archives orales située au cœur du projet de matrimonialisation de la BD initié par Les Bréchoises, implique la prolongation d'autres rapports aux savoirs, notamment les savoirs situés. Saisissant l'histoire de la bande dessinée, depuis une approche féministe et complexifiée, on peut aborder les rapports de pouvoir qui ont structuré cette industrie et qui ont conduit à l'invisibilisation, voire à l'exclusion d'un grand nombre de ses acteurices. Je n'ai pas seulement voulu montrer comment le manque de documentation sur certain.e.s professionnel.le.s et groupes sociaux a contribué à leur invisibilisation. J'ai aussi voulu montrer les processus de réhabilitation d'un 
matrimoine qui est loin d'être inexistant. Dès lors, il ne me reste plus qu'à continuer à étudier les constellations créatrices actuelles, pour rendre compte des lignées dans lesquelles elles peuvent s'inscrire. S'interroger sur l'absence des femmes bédéastes dans la recherche universitaire m'a fait me rapprocher de l'histoire culturelle, pour ensuite la questionner. Puisque les femmes sont actives dans ce secteur depuis sa création, j'interroge de manière critique l'exclusion vécue par ces créatrices et non leur absence supposée. Avoir conscience de ce constat et la possibilité de le transformer au travers de ce projet d'archives des femmes dans la BD, n'est que la suite logique d'une recherche qui espère revêtir tout son sens pratique et social.

\section{BIBLIOGRAPHIE}

AQUATIAS, Sylvain. 2018. " Genre et légitimité dans l'édition de bande dessinée - partie 1 », Comicalités ; Culture graphique : créations et représentations, [En ligne], consulté le 19 avril 2021. URL : http://journals.openedition.org/comicalites/2639 ; DOI : https://doi.org/10.4000/comicalites. 2639.

ARNOLD, Markus. 2021. «L'archive dans la bande dessinée contemporaine du tournant postcolonial », conférence (sans publication) à l'université Sorbonne Nouvelle.

DESCAMPS, Florence. 2019. Archiver la mémoire : de l'histoire orale au patrimoine immatériel. Collection Cas de figure 49. Paris, Éditions EHESS.

DESPRET, Vinciane. 2010. " "L'effet sans nom" : l'anonymat dans les pratiques de la psychologie », Vinciane Despret, d'un texte à l'autre..., consulté le 19 avril 2021. URL : www.vincianedespret.be/2010/04/leffet-sans-nom/.

DÉTREZ, Christine. 2018. « L'exclusion genrée, un jeu en trois manches », in Sylvie Octobre et Frédérique Patureau, Normes de genre dans les institutions culturelles, Paris : Presses de la Fondation Nationale des sciences politiques.

FARGE, Arlette. 1997. Le goût de l'archive, Paris : Éd. du Seuil, 1997, p. 70.

FAVRET-SAADA, Jeanne. 2020 [1977]. Les mots, la mort, les sorts, Paris : Gallimard.

GONZÁLEZ, José. 2019 [2017]. « Archives et contre-archives minoritaires : élites et discours subalternes ", CEIIBA (Centre d'études ibériques et ibéroaméricaines - cultures romanes et amérindiennes), consulté le 19 avril 2021. URL : https://ceiiba.hypotheses.org/86.

HERTIMAN, Marys R. 2020. “ “Il n'y a pas des liens entre la notoriété des femmes et [leurs] traces...”. Entretien avec Isabelle Bastian-Dupleix et Caroline Raynaud, commissaires de l'exposition C. Meurisse (BPI) », L'Exporateur littéraire, consulté le 19 avril 2021. URL : https:// www.litteraturesmodesdemploi.org/entretien/il-ny-a-pas-des-liens-entre-la-notoriete-desfemmes-et-leurs-traces-entretien-avec-isabelle-bastian-dupleix-et-caroline-raynaudcommissaires-de-lexposition.

HIRAUX, Françoise \& MIRGUET, Françoise (dir.). 2013. Les Archives personnelles. Enjeux, acquisition, valorisation, Paris : Academia-L'Harmattan. 
KOHN, Jessica. 2018. «Travailler dans les Petits Mickeys » : les dessinateurs-illustrateurs en France et en Belgique de 1945 à 1968. Thèse de doctorant en Histoire. Paris : Université Sorbonne Paris Cité. [En ligne], consulté le 19 avril 2021. URL : https://tel.archives-ouvertes.fr/tel-02147283.

MILQUET, Sophie \& REYNS-CHIKUMA, Chris. 2016. « Préface au numéro spécial », ALTERNATIVE FRANCOPHONE [En ligne], Vol. 1 No. 9 : « La bande dessinée au féminin/Female Comics », consulté le 19 avril 2021. URL : https://journals.library.ualberta.ca/af/index.php/af/article/view/27188/ pdf.

NOCÉRINO, Pierre. 2021. «Les ateliers partagés : entre professionnalisation et politisation des auteurs et autrices », Neuvième art 2.0, dossier : « La vie d'atelier » [En ligne], consulté le 19 avril 2021. URL : neuviemeart.citebd.org/spip.php?article1355.

RUIZ, Émilien. 2011. « Pourquoi s'intéresser à l'historiographie? », Devenir historien-ne, consulté le 19 avril 2021. URL : https://devhist.hypotheses.org/34.

SCOTT, Joan W. 2020. Théorie critique de l'histoire. Identités, expériences, politiques, Paris : Fayard.

TIREHOTE-CORBIN Maëlys. 2019. Enquête en école de bande dessinée. La fabrique des créateurices, un apprentissage genré ?, Mémoire de Master, sous la direction de D. Naudier, EHESS, Paris

« Histoire », Le Robert, [En ligne], consulté le 19 avril 2021. URL : https://

dictionnaire.lerobert.com/definition/histoire.

Centre Nationale de Ressources Textuelles et Lexicales - CNRTL, [En ligne], consulté le 19 avril 2021 URL : https://www.cnrtl.fr/definition/histoire.

« Mémoire », Centre Nationale de Ressources Textuelles et Lexicales - CNRTL, [En ligne], consulté le 19 avril 2021. URL : https://www.cnrtl.fr/definition/m\%C3\%A9moire.

Association Mnémosyne. 2005. «La bande dessinée : un nouveau chantier pour l'histoire des femmes et du genre ». Journée d'études [En ligne] consulté le 19 avril 2021. URL : https:// www.mnemosyne.asso.fr/mnemosyne/journee-detudes-2005/

\section{NOTES}

1. À ce propos, je renvoie aux déclarations de Franck Bondoux faites lors d'un entretien dans l'émission Le Grand Journal de Canal+: «Le Grand Prix, c'est un prix qui récompense un auteur de bande dessinée - ou une auteure... pourquoi pas - pour l'ensemble de son cuvre. Clairement, sans les vexer, les derniers lauréats, c'est Willem, c'est Bill Watterson, c'est Otomo, donc des auteurs qui ont un certain âge, une cuvre, aussi. Et la vérité nous oblige à dire que quand on remonte dans l'histoire de la bande dessinée, par exemple dans les publications que nous connaissons tous, Tintin, Spirou, Pilote, (À suivre), Pif gadget, il y a très, très, très peu de femmes et qu'elles se comptaient sur les doigts d'une main. Il ne faut pas qu'on fasse dans l'art de la discrimination positive, qui n'a pas lieu d'être. C'est-à-dire qu'on ne va pas mettre des femmes pour le fait de mettre des femmes. Ce ne serait pas bien, si elles étaient lauréates. » Cité dans : Gilles Ciment, «Femmes dans la bande dessinée, des pionnières à l'affaire d'Angoulême (8/12): Angoulême, acte 2, les “"nhomminations"' pour le Grand Prix ", site de l'Association Artémisia, publié le 18 janvier 2017. URL : www.assoartemisia.fr/femmes-dans-labande-dessinee-angouleme-acte-2-nhomminations-grand-prix/, consulté le 13 décembre 2021.

2. Les recherches de Maëlys Tirehote-Corbin ont montré que « sur les 37 ouvrages imposés dans des cours de BD, 3 ont été écrits par des femmes (Cestac, Brétecher et Goetzinger) 》 (TirehoteCorbin, $2019:$ 47-48).

3. Comme ceux de Jessica Kohn et de Pierre Nocérino ou des enquêtes des États généraux de la BD de 2016. 
4. L'une des premières esquisses d'historicisation du travail des créatrices de BD en France se trouve dans la série d'articles «Histoire de la BD féminine ", signée par Pierre Couperie entre 1976 et 1978 dans le magazine Ah! Nana. Si ces articles se présentent avant tout comme des portraits d'autrices (venant des multiples endroits du monde), il s'agit d'une des premières traces d'inscription des femmes dans l'histoire du neuvième art.

5. Sans se centrer sur les disparités professionnelles entre les artistes-auteurices, la thèse de Pierre Nocérino, offre quelques données pouvant fournir de la matière à de nouvelles recherches autour des rapports genrés de pouvoir.

6. Le master de Maëlys Tirehote-Corbin, permet de rendre compte des difficultés rencontrées par les femmes pour se former aux métiers de la bande dessinée et notamment pour trouver une place dans le monde professionnel. Doctorante en sociologie à l'Université de Lausanne, les recherches de Maëlys Tirehote-Corbin portent aujourd'hui sur « La féminisation des métiers de la bande dessinée ».

7. Claire Bretécher, Chantal Montellier, Florence Cestac, Jeanne Puchol, Marjane Satrapi, Catel, Anne Goetzinger, Lisa Mandel, Manon Iessel, Marie-Mad, Pénélope Bagieu et Catherine Meurisse.

8. Les Bréchoises sont constituées par les chercheuses Sophie Bonadè, Laura Caraballo, MariePaul Noël (créatrice de BD), Maëlys Tirehote-Corbin, Johanna Schipper (chercheuse, enseignante et créatrice de $\mathrm{BD}$ ) et moi-même.

9. Toutefois, la précision même de cette terminologie juridique est biaisée puisque les traducteurices elleux aussi touchent un pourcentage (variable), en droits d'auteurices, mais iels n'ont pas été comptabilisés dans l'enquête «Auteurs » des EGBD ni dans le comptage fait par Gilles Ratier pour l'ACBD.

10. Environ 6 dans les livres spécialisés et autour de $12 \%$ dans les dictionnaires de la BD (sachant que Gilles Ratier et Patrick Gaumer collaborent ensemble à plusieurs reprises, notamment au sein de l'association Artémisia).

11. La liste, non mise à jour, peut être consultable tweet publié en mars 2021 et disponible sur le lien suivant: https://twitter.com/MarysRGH/status/1371137980698660868?s=20, consulté le 13 décembre 2021.

12. L'un de rares gisements documentaires sur le matrimoine de la BD française, a été conçu par Élise Ponce qui, dans une série de podcasts intitulée Auriculaire, a interrogé 24 créatrices de BD sur leur vie professionnelle et l'inscription de leur production dans leur constitution identitaire. En ce qui concerne la recherche scientifique, l'un des premiers travaux portant sur les professionnel.le.s de la bande dessinée était celui de Jessica Kohn. Sa thèse en histoire, soutenue en 2018, qui portait sur l'ensemble des dessinateurices de bande dessinée, les noms d'un groupe des femmes dans cette industrie (en France et en Belgique) entre 1945 et 1968.

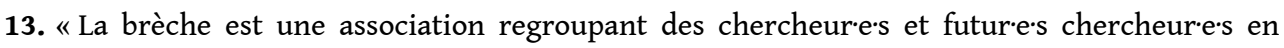
bande dessinée. Son but est de produire et diffuser de la recherche académique sur la bande dessinée, faciliter les rencontres entre chercheur·es, et explorer également le médium de la bande dessinée pour créer du savoir. » Présentation de l'association sur leur site. URL : https:// labrechebd.com/, consulté le 13 décembre 2021.

14. Plus de détails sur ce projet sur le site de l'école universitaire de recherches ArTeC: https:// eur-artec.fr/projets/creatrices-de-bandes-dessinees/, consulté le 13 décembre 2021.

15. Une des principales difficultés liées à cette enquête consistait à la collecte des témoignages anonymisés ou nominalisés. Néanmoins, répondre aux contraintes de l'ethnographie classique aurait été contraire à notre mission de visibilisation. À propos des l'impact des pratiques d'anonymisation dans la recherche, je renvoie à l'article de Virginie Despret " "L'effet sans nom" : l'anonymat dans les pratiques de la psychologie ", publié dans son carnet de recherches, le 28 avril 2010 [En ligne]. URL: http://www.vincianedespret.be/2010/04/leffet-sans-nom/, consulté le 13 décembre 2021. 
16. Détermination du budget (défraiement des participants, avantages en nature [prêt de salles, matériel d'enregistrement/captation], achat des boîtes et d'autres matériels d'archive, etc.) et des modes de financement.

17. Les premières créatrices convoquées sont en lien direct avec nos recherches personnelles en cours. Ceci nous permettait de mutualiser nos recherches avec ce projet extérieur.

18. Ces derniers arguments sont de taille puisque, dans le travail sur archives, il nous a été très difficile de convaincre la plupart des créatrices de l'importance (historique et sociale) que revêtent certaines sources telles que les journaux intimes.

19. À ce propos, voir les travaux suivants : Florie Boy (2009), «Les femmes dans la bande dessinée d'auteur depuis 1970. Itinéraires croisés: Claire Brétecher, Chantal Montellier et Marjane Satrapi »; Bérénice Motais de Narbonne (2016), «La Pilule Rouge, les femmes dans la BD »; Virginie Talet (2004) « Métal Hurlant et Ah! Nana : deux expériences dans l'univers underground en France, 1975-1987 », et Blanche Delaborde (2005), « Ah ! Nana (1976-1978) ». Histoire.

20. L'histoire qui les concerne, l'histoire qu'iels vont raconter, l'histoire sociale qu'iels ont vécue et entendue, etc.

21. Par historiographie, j'entends de la pratique de l'analyse et de l'écriture de l'histoire. Toutefois, il me paraît nécessaire de renvoyer vers Marie-Paule Caire-Jabinet (citée par Ruiz 2011), expliquant que ce terme « apparaît au XIXe siècle [... et] signifie selon les cas : l'art d'écrire l'histoire, la littérature historique, ou encore “'l'histoire littéraire des livres d'histoire”' (Littré, 1877) ».

22. D'après Florence Descamps (2019: 160-161), les archives (orales) de type «conservatoires visent à fixer et à stocker, à titre préventif ou en urgence, des informations, des récits ou des souvenirs, mais sans objectif d'exploitation déterminé ; [...] [les archives] monumentaires, visent à fixer et à conserver la mémoire culturelle d'un événement, d'une expérience ou d'une communauté, comme une butte-témoin [...] [et les archives] transmissives, visent à transmettre des savoirs ou des savoir-faire ".

23. Dans le cas des autrices de bande dessinée, beaucoup de leurs crayonnés (dessins plus au moins achevés, avant la mise en couleur et souvent sans l'inscription du texte) et de leurs originaux sont vendus, pour en faire des compléments financiers, ce qui fait éclater un fonds propre à un.e artiste-auteurice. Par ailleurs, les déménagements successifs ou le manque de place conduisent souvent à la perte ou la destruction des sources pouvant constituer leur fonds d'archives.

24. Sur ces questions, voir : Claire Delahaye, "L'archive et le politique : enjeux et perspectives ", Revue française d'études américaines, vol. 162, no. 1, 2020, pp. 3-19; Fabiana De Souza, "Réactualiser l'archive, réécrire l'histoire. Des pratiques artistiques décoloniales ", REVUE Asylon(s), $\mathrm{N}^{\circ} 15$, février 2018, «Politique du corps (post) colonial», [En ligne], URL : http:// www.reseau-terra.eu/article1406.html, consulté le 13 décembre 2021; Sémir Badir et Baetens Jan, « Présentation : L'héritage et l'archive. » Protée, volume 32, numéro 2, automne 2004, p. 5-8. https://doi.org/10.7202/011167ar, consulté le 13 décembre 2021 et Vincent Duclert, «Les historiens et la crise des archives ", Revue d'histoire moderne \& contemporaine, vol. no48-4bis, no. 5, 2001, pp. 16-43.

25. Dans mes entretiens avec Jeanne Puchol et Chantal Montellier, considérées comme quelquesunes de pionnières de la $\mathrm{BD}$, ces autrices ont insisté sur cette question qui les a constamment été posées « qu'est-ce que ça fait d'être une femme dans un milieu peuplé d'hommes? ». N'ayant que très rarement eu l'occasion de collaborer avec d'autres consœurs, elles avaient intégrées leur proportion minoritaire, pour ne pas dire leur rareté, dans l'industrie de la BD. Par ailleurs, et toujours à ce propos, Lisa Mandel avait organisé en 2016 une table ronde critique, où elle demandait à un groupe d'auteurs ce que ça signifie d'être un homme dans la BD. Cet événement, devenu culte depuis, a été filmé et est accessible sur le site du Collectif des créatrices de BD 
contre le sexisme. URL : https://bdegalite.org/les-hommes-et-la-bd/, consulté le 13 décembre 2021.

26. L'éditrice, traductrice et scénariste Marie-Paule Noël a récemment fait part de son sentiment de ses difficultés concernant son statut dans l'industrie de la BD. Même si elle travaille dans la BD depuis 1997, c'est son premier contrat signé (2021) en tant que scénariste, qu'elle peut «enfin pouvoir clamer partout qu[elle est] dorénavant une "vraie" autrice de BD ». Marie-Paule Noël, publié sur Twitter le 23 juin 2021. URL: https://twitter.com/mpnoel/status/ 1407631813850316803 ?s=20, consulté le 30 juin 2021.

27. Dans le secteur jeunesse ou la presse catholique pour la plupart.

28. Afin de pouvoir repérer les autrices de BD ayant publié en France chaque année, de 2010 à 2019, j'ai dépouillé les listes des livres assimilés aux bandes dessinées vendus en France. Ces listes ne comptaient pas moins de 3000 références, qu'il fallait par la suite vérifier. Par exemple, rien que pour l'année 2018, après avoir supprimé les mentions des mangas, comics et livres jeunesse, mon comptage signalait 1139 nouveautés (BD franco-belge et italienne, humour, roman graphique, histoire et politique) dont seule 180 mentionne la part auctoriale des femmes. De cette résultante, seulement 64 albums ont été signés uniquement par des femmes françaises ou installées en France.

29. La problématique de ma recherche doctorale porte sur la praxis discursive des autrices de BD. Elle est comprise comme un acte sur lequel pèsent des contraintes (Judith Butler). Elle n'est pas spontanée puisque son expression n'est jamais naturelle ni va de soi, il s'agit d'une pratique réfléchie qui signalerait non seulement la puissance d'agir qui traverse le discours, mais aussi les rapports de force et de pouvoir qui le structurent.

30. À ce sujet, voir Jean-Clément Martin, «Histoire, mémoire et oubli pour un autre régime d'historicité ", Revue d'histoire moderne \& contemporaine, 2000/4 (no 47-4), p. 783-804. DOI : 10.3917/rhmc.474.0783. URL : https://www.cairn.info/revue-d-histoire-moderne-etcontemporaine-2000-4-page-783.htm, consulté le 13 décembre 2021; François Dosse, «Entre histoire et mémoire : une histoire sociale de la mémoire ", in : Raison présente, $\mathrm{n}^{\circ} 128,4 \mathrm{e}$ trimestre 1998. Mémoire et histoire. pp. 5-24, François Cadiou, Anne Lemonde, Clarisse Coulomb et al., « IX / Histoire et mémoire ", in : François Cadiou éd., Comment se fait l'histoire. Pratiques et enjeux. Paris, La Découverte, «Repères », 2011, p. 163-178. URL : https://www.cairn.info/comment-sefait-1-histoire--9782707169174-page-163.htm, consulté le 13 décembre 2021 ; ou Patrick-Michel Noël, « Entre histoire de la mémoire et mémoire de l'histoire : esquisse de la réponse épistémologique des historiens au défi mémoriel en France ", Conserveries mémorielles [En ligne], \#9 | 2011, mis en ligne le 15 avril 2011, URL: http://journals.openedition.org/cm/820, consulté le 13 décembre 2021.

31. Les travaux de Sylvain Lesage offrent un vaste panorama sur l'histoire et la patrimonialisation de la BD franco-belge, notamment en tant que dispositif et objet médiatique. Cf. : « De la presse qui ne se lit que d'une main au panthéon du 9e art : Barbarella, de Jean-Claude Forest ». Les petits aventuriers du quotidien. Bande dessinée, journal et imaginaires médiatiques (XIXe-XXIe siècles), Guillaume Pinson; Alexis Lévrier, juin 2017, Reims, France ; «Les archives Casterman : un continent inconnu », Strence ${ }^{\circ} 11$, «La collection, fabrique éditoriale des œuvres pour la jeunesse : l'apport des archives » (dossier : « Fonds d'archives et collections »), oct. 2016, https://strenae.revues.org/1623 (consulté le 13 décembre 2021) ou encore «Les archives Casterman : un continent inconnu ", Strenae - Recherches sur les livres et objets culturels de l'enfance, Association Française de Recherche sur les Livres et les Objets culturels de l'Enfance (AFRELOCE), 2016.

32. Beaucoup de collections sont restées inachevées ou ont changé de maison d'édition; des planches d'une même œuvre ou d'un.e même auteurice peuvent se retrouver dans plusieurs endroits différents suite à leur vente dans des galeries ; les premières publications n'étaient pas enregistrées, identifiées, voire indexées (il en va de même avec la production des fanzines, même 
aujourd'hui, qui n'ayant pas un accompagnement éditorial, sont autoproduits et ne bénéficient pas d'un numéro de référenciation.

33. Le séminaire «Décoloniser le patrimoine. Critical heritage studies. Épistémologies, réception et actualité (Europe, Asie, Monde)» de l'Institut National du Patrimoine, s'est penchée la dimension politique des rapports genrés au travers des legs. Les séances, enregistrées, sont consultables depuis la chaîne podcast dédié: https://soundcloud.com/institut-national-dupatrimoine/sets/decoloniser-le-patrimoine, consulté le 13 décembre 2021. Depuis 2015, l'association HF Île-de-France investit l'espace public et organise une série d'événements culturels ayant pour objectif la visibilisation des femmes du passé et leur héritage.

34. Restitution qui passe par la collecte d'archives de diverses natures appartenant aux premières créatrices de $\mathrm{BD}$, encore en vie, résidant en France.

35. Marie Sorbier, «Le matrimoine n'est pas un néologisme, mais un mot effacé par l'Histoire », Affaire en cours, émission de France Culture, le 29 janvier 2021 [En ligne], URL: https:// www.franceculture.fr/emissions/affaire-en-cours/affaires-en-cours-du-vendredi-29-

janvier-2021, consulté le 13 décembre 2021. Le site de l'association Le matrimoine, propose différentes sources pour s'informer sur les legs des femmes, mais aussi sur la masculinisation des expressions, effaçant et invisibilisant les femmes de l'histoire. Lien permettant d'accéder à cette association : https://www.lematrimoine.fr, consulté le 13 décembre 2021.

\section{RÉSUMÉS}

L'absence présumée des créatrices de bande dessinée dans l'histoire de ce médium résulte, en partie, du manque de sources pouvant conduire à la restitution de leurs traces. Dans ce contexte, un groupe de chercheuses, se faisant appeler Les Bréchoises, se sont fédérées afin de provoquer les archives des femmes dans le neuvième art français et ainsi constituer des monuments voulus, des monuments-mémoire. Cet ambitieux projet de recherche rend compte d'une posture ambivalente : scientifique et militante à la fois. Dès lors, en partant de l'inexistence présupposée du matrimoine de la BD, et du dévoilement des coulisses d'un projet de mise en mémoire, cet article revient sur les composants de la pratique organique de l'archive.

The presumed absence of female comic strip creators in the history of this medium is due, in part, to the lack of sources that could lead to the restitution of their traces. In this context, a group of women researchers, calling themselves Les Bréchoises, have joined forces to create archives of women in the French ninth art and thus to constitute desired monuments, monuments of memory. This ambitious research project reflects an ambivalent posture: scientific and militant at the same time. Starting from the presupposed non-existence of the comic book heritage and the unveiling of the backstage of a project of memorialization, this article returns to the components of the organic practice of the archive. 


\section{INDEX}

Thèmes : Explorations

Mots-clés : matrimoine, archives, créatrices de bande dessinée, mise en mémoire, réécriture de l'histoire

Keywords : archives, women cartoonists, tracing, matrimony, rewriting history

\section{AUTEUR}

\section{MARYS RENNÉ HERTIMAN}

Université Paris 8

Marys Renné Hertiman est chercheuse-doctorante en information et communication (ED 401, Sciences sociales, Université Paris 8), rattachée au Centre de recherche interuniversitaire, expérience, ressources culturelles, éducation (EXPERICE). Sa thèse porte sur les « Discours des femmes dans la BD française contemporaine ». Avec l'équipe de chercheuses Les Bréchoises, elle coordonne le projet «Créatrices de bandes dessinées : histoire, mémoire, revendications et représentations des femmes dans le 9e art ». Publications récentes : « La valorisation du travail des femmes dans la bande dessinée : entre médiation, remédiation et mobilisation ", RELIEF Revue Électronique de Littérature Française, 14(2), décembre 2020 ; « La socialité du discours andersennien », revue Deshima n 13, janvier 2020 et «Les Sans-voix ou les Misérables d'HansChristian Andersen », revue Signes, Discours et Sociétés, n² 20, novembre 2019. 